\title{
La altura histórica de Polo en la filosofía
}

\author{
Polo's height in the history of philosophy \\ Juan Fernando Sellés \\ Universidad de Navarra (España)
}

Recibido: 11.09.2019

Aceptado: 18.09.2019

\section{RESUMEN}

La altura histórica de un pensador y de una corriente de filosofía se mide por los actos cognoscitivos que principalmente ejerce y por los actos reales que descubre. $\mathrm{Ni}$ unos ni otros son irrestrictos, pero siempre cabe ejercer más los primeros y descubrir más de los segundos. Polo ha descubierto la jerarquía de unos y otros y ha puesto orden jerárquico entre los diversos pensadores precedentes. Esto le coloca en la cima de la historia de la filosofía, pero como ésta no cierra, ¿cómo proseguirle?

\section{PALABRAS CLAVE \\ JERARQUÍA NOÉTICA Y REAL; POLO; CUMBRE; PROSECUCIÓN}

\begin{abstract}
The historical height of a thinker and a current of philosophy is measured by the cognitive acts that it mainly exercises and by the real acts it discovers. Neither are unrestricted, but we can always exercise more first and discover more of the latter. Polo has discovered the hierarchy of one and the other and has put hierarchical order among the various preceding thinkers. This places him at the top of the history of philosophy, but since it does not close, how can we continue?

KEYWORDS

NOETIC AND REAL HIERARCHY; POLO; SUMMIT; PROSECUTION
\end{abstract}

\section{Planteamiento}

LA SUPERIOR O INFERIOR ALTURA DE UNA CORRIENTE DE FILOSOFÍA, Y aSimismo de un pensador en ella enmarcado, depende, por una parte, del nivel cognoscitivo que ocupan las operaciones racionales que predominantemente ejerce, pues éstas pertenecen a una determinada franja de la am- 
plia escala del conocer humano y, por otra, de los temas reales conocidos por dichos niveles. A tal nivel Polo lo llamaba "altura histórica» ${ }^{1}$, y aquí se busca cual es la altura de Leonardo Polo en la historia de la filosofía.

En una primera aproximación hay decir que en el hombre cabe distinguir cuatro dimensiones naturales en su conocer, que de inferior a superior son: el conocer sensible, el racional, el intelectual y el personal. Es claro que cada una de ellas admite pluralidad de niveles. Así, en el nivel sensible, es patente que los sentidos internos son más cognoscitivos que los externos; en el racional, que los hábitos adquiridos lo son más que las operaciones inmanentes; en el intelectual o propio de los hábitos innatos, la sabiduría es más cognoscitiva que el hábito de los primeros principios, y éste que la sindéresis. Por último, en el nivel del intelecto personal cabe hablar de búsqueda del Origen de la persona que uno es y de búsqueda de Destinatario, y ésta última es superior a la anterior ${ }^{2}$.

Pues bien, es claro que no todas las corrientes de filosofía y sus representantes han ejercido en su trabajo filosófico todos los niveles aludidos ${ }^{3}$, sino que normalmente se han ceñido preferentemente a un determinado nivel al que han dado más importancia que a los demás. Por ejemplo: cabe sostener que los empirismos, sensismos, utilitarismos o materialismos de todos los tiempos han primado al conocer sensible por encima del resto (esto no indica, obviamente, que no hayan ejercido el racional, pues han defendido 'racionalmente' la supuesta hegemonía del conocer sensible). No menos

1 «Suelo hablar de altura histórica, que es algo así como el emplazamiento en que uno se encuentra y desde el cual se orienta respecto de los hallazgos filosóficos logrados hasta hoy, a partir del convencimiento de que la filosofía nunca está terminada». Polo, L., Antropología trascendental, I. La persona humana, en Obras Completas, Serie A, vol. XV, Pamplona: Eunsa, 2015, 23.

2 Por lo demás, los cuatro niveles y cada uno de sus respectivos actos cognoscitivos pueden ser elevados sobrenaturalmente: los sentidos por revelaciones sensibles; la razón, por la virtud infusa de la prudencia y por el don de ciencia; el intelecto, por los dones superiores del Espíritu Santo (la sindéresis, por el don de consejo; el hábito de los primeros principios por el don de entendimiento; el hábito de sabiduría por el don de sabiduría); el intelecto personal, aquí por el lumen fidei y allá por el lumen gloriae.

3 «No todos los hombres ni todas las épocas ejercen todas las operaciones intelectuales, ni tampoco en el comienzo de la filosofía se ejercieron. Asimismo, de las operaciones intelectuales y de sus hábitos dependen las disciplinas teóricas que tienen también un comienzo histórico preciso. En suma, la historia de la filosofía puede formularse desde la axiomática propuesta para la teoría del conocimiento». Polo, L., Curso de teoría del conocimiento, vol. II, en Obras Completas, Serie A, vol. V, Pamplona: Eunsa, 2015, 227. 
claro es que los racionalismos, idealismos o la fenomenología, por poner otros ejemplos, se han ceñido sobre todo a la operatividad racional teórica, mientras que otras corrientes como el pragmatismo, buena parte de la filosofía analítica y la hermenéutica se han ajustado más a la operatividad racional práctica. Por otra parte, no han faltado entre los pensadores clásicos griegos y medievales los que, tras distinguir entre la razón (ratio, logos) y el intelecto (intellectus, noûs) -o también entre la razón inferior (ratio inferior) y la superior (ratio superior) - y tras haber defendido que ésta es superior a aquélla, han sacado más partido a la primera que a la segunda.

Obviamente, estudiar en qué nivel noético se ha movido preponderantemente cada filósofo o cada escuela filosófica daría para un trabajo enciclopédico, aquí completamente improcedente. Pero, a mi modo de ver, nadie salvo Leonardo Polo, ha descubierto 'explícitamente' que el 'conocer personal' humano está a nivel de acto de ser y, por tanto, que es el nivel noético natural superior humano, y en consecuencia, que excede a los demás ${ }^{4}$. A esto cabe añadir que nadie, salvo él, lo ha ejercido con suficiencia para mostrar mejor su tema propio: el ser pluripersonal divino.

Si se admite que el conocer humano es jerárquico -asunto fácil de admitir, si se tiene la calma suficiente para notar que esta tesis se puede traducir axiomáticamente-, y si se descubren con suficiencia sus diversos niveles -asunto tan arduo que la mayoría de los pensadores no lo ha llevado a cabo-, cabe sentar de modo sencillo que Polo está en la cúspide de la historia de la filosofía por lo que a la noética se refiere. Ahora bien, para mostrar que esta afirmación no obedece a una mera opinión subjetiva, tendríamos que proceder pacientemente a desplegar un exhaustivo curso de teoría del conocimiento -cabe decir de paso que el de Polo es el más amplio y riguroso habido hasta la fecha ${ }^{5}$. Pero la precedente tarea es aquí,

4 Aunque en esa dirección trabajaron Aristóteles, San Albero Magno, Teodorico el Teutónico y Francisco Canals, sólo Polo y sus discípulos han defendido con suficiencia la existencia del conocer personal y su superioridad sobre el resto. Cfr. al respecto mi trabajo: $E l$ intelecto agente y los filósofos. Venturas y desventuras del supremo hallazgo aristotélico sobre el hombre (I), ss. IV a. C. - XV, Eunsa, Pamplona, 2012; (II), ss. XVI-XVII, Eunsa, Pamplona, 2017; (III), ss. XVIII-XXI, Pamplona: Eunsa, 2017.

5 Cfr. Polo, L., Curso de teoría del conocimiento, vols. I-IV, en Obras Completas, Serie A, vols. IV-VII, Pamplona: Eunsa, 2015-2019; El acceso al ser, en Obras Completas, Serie A, vol. II, Pamplona: Eunsa, 2015; Nominalismo, idealismo y realismo, en Obras Completas, Serie A, vol. XIV, Pamplona: Eunsa, 2016; El conocimiento del universo físico, en Obras Completas, Serie A, vol. XX, Pamplona: Eunsa, 2015; Epistemología, creación y divinidad, en Obras Completas, Serie 
obviamente, inabordable.

Si Polo no es superior a los demás pensadores, no ha ejercido más que los otros los conoceres superiores humanos: el hábito de sabiduría y el personal; y consecuentemente, no ha perfilado más que otros sus temas propios, los cuales ocupan la cúspide de lo real: la propia realidad personal humana y pluripersonal divina, respectivamente. Negar esto equivale a decir que, o bien no existen el hábito de sabiduría y el conocer personal, o que la antropología trascendental poliana y la teología natural que ella posibilita son novelas, es decir, no responden a hallazgos. Sin embargo, nadie -de momento- las ha refutado. Se puede alegar al respecto que Polo es tan reciente como desconocido. Pero si sus propuestas no presentan contradicción con las de los grandes filósofos del pasado ni ha sido refutado por los actuales, al menos hay que darles un voto de confianza de cara al futuro.

\section{LA FILOSOFÍA PROGRESA EN LA MEDIDA EN QUE SE EJERCEN ACTOS SUPERIORES DE CONOCER Y SE DESCUBREN CON ELLOS SUPERIORES ACTOS DE SER}

Explicitemos ambas tesis:

\section{I.1. LA FILOSOFÍA PROGRESA EN LA MEDIDA EN QUE SE EJERCEN ACTOS SUPERIORES DE CONOCER}

Esto indica que la mejora en filosofía no es serialmente progresiva ${ }^{6}$ (aunque conviene leerla siguiendo su serie temporal ${ }^{7}$ ), pues si unos pensa-

\footnotetext{
A, vol. XXVII, Pamplona: Eunsa, 2015. Junto a esos libros, clásicos en el autor para esta materia, hay alusiones a los diversos niveles del conocer humano desperdigados en todas las obras polianas.

6 «La historia no es un proceso sucesivo de fases que abren otras y quedan como un precedente. Hay razones para pensar que no es así, que 'la sucesión sucesiva de sucesos sucedidos en los sucesivos siglos' es una torpe estupidez». Polo, L., Curso de psicología general, en Obras Completas, Serie A, vol. XXI, Pamplona: Eunsa, 2018, 293.

7 «Hay que empezar a leer y enterarse de lo que piensan los primeros, hay que seguir la línea de la historia de la filosofía. Empezar con Kant, Hegel o Heidegger está mal, porque lo que dicen depende de lo que han dicho los anteriores. Si en filosofía no se sigue su historia, no se va con buen pie. Si uno no entiende a Parménides ni a Tales de Mileto, el resto de la filosofía no la entiende. A grandes rasgos esto es lo que aparece en la Introducción a la filosofía, cómo se debe leer la filosofía». Polo, L., Conversaciones en Torreblanca (Colombia), pro manuscripto, 1996, 12.
} 
dores posteriores ejercen actos de conocer inferiores a los precedentes, sus filosofías tendrán menos alcance que la de los anteriores.

Los filósofos siempre han ejercido diversos actos de conocer, pero no en todos los tiempos se ha sabido cuál es la índole de un acto de conocer, sino más bien en $\operatorname{pocos}^{8}$. Bien mirado, ese olvido marca un declive en la historia de la filosofía, porque asimila el acto a la realidad del movimiento y torna temporalista la temática filosófica. Además, como los hábitos adquiridos son superiores a las operaciones inmanentes, los periodos de la historia del pensamiento en que éstos están olvidados son asimismo de decrecimiento $^{9}$, y aquellos otros en los que se considera que los hábitos adquiridos son menos cognoscitivos que los actos, son periodos reductivos ${ }^{10}$. Por su parte, los hábitos innatos (no a la inteligencia como potencia, sino al intelecto en acto o intelecto agente ${ }^{11}$, en rigor, al acto de ser personal ${ }^{12}$ ) son superiores a

8 «En la historia de la filosofía ha sufrido un largo eclipse el tema de los movimientos discontinuos, de las operaciones inmanentes... La razón fundamental simplemente es ésta: hay un momento en el que se empieza a sospechar que no hay operación cognoscitiva, o que el conocimiento es pasivo. En la sospecha intervienen el intuicionismo y el voluntarismo. Esto tiene lugar en el siglo XIV». Polo, L., Curso de teoría del conocimiento, vol. I, en Obras Completas, Serie A, vol. IV, Pamplona: Eunsa, 20189, 75-76.

9 «En los planteamientos modernos la noción de hábito está perdida. Triste pérdida que hace a la filosofía tan objetualista, y además, que sean tan frecuentes la conculcación del axioma A (a saber, 'El conocimiento es acto') y la apelación a la intuición o a la construcción del principio de conciencia, cuya entraña es el voluntarismo, es decir, una confusión entre dos órdenes de actividad». Polo, L., Curso de teoría del conocimiento, vol. II, ed. cit., 184. $10 \ll \mathrm{El}$ conocimiento de los primeros principios es habitual y no objetivo... el hábito citado es un acto intelectual superior a cualquier operación... La filosofía tradicional (tomista) no admite la superioridad del conocimiento habitual sobre el objetivo, sino que sostiene que el conocimiento objetivo es más claro que el habitual». Polo, L., Antropología trascendental, ed. cit., 133.

11 «La existencia de hábitos superiores a los adquiridos está reconocida en la filosofía tradicional. Los llamados hábitos innatos se caracterizan precisamente por no ser perfectivos de la potencia intelectual, es decir, por añadirse a ella de un modo distinto a los hábitos que derivan de su ejercicio operativo. En otro caso no cabría decir que la inteligencia es una tabula rasa, pues todos los actos cognoscitivos son temáticos. Además, los hábitos innatos no requieren una operación previa. Por consiguiente, los hábitos innatos dependen del intelecto agente. En la tradición se admiten tres hábitos innatos: el hábito de los primeros principios o intellectus principiorum - que es el más estudiado-, el hábito de sabiduría y la sindéresis». Polo, L., Antropología trascendental, ed. cit., 177.

12 «La denominación de 'hábito innato' no es completamente acertada. La uso para recalcar que su sede no es la potencia. Pero en cuanto que proceden de la persona son, 
los adquiridos, y el conocimiento explícito de ellos está todavía más oculto en la historia de la filosofía, lo cual indica -con más motivo aún que en el caso del olvido de los precedentes niveles noéticos- que los periodos en que éstos están desconocidos no son álgidos. Por su parte, el conocer personal es superior a los precedentes, pero solo excepcionalmente ha sido tenido en cuenta en la historia de la filosofía.

A lo que precede se podría objetar que los filósofos posteriores lo tienen fácil para superar a los anteriores, puesto que cuentan con su legado, lo pueden meditar pacientemente y lo pueden superar hasta ver más que ellos. Sin embargo, históricamente no ha sido así, al menos por dos motivos: Uno, porque buena parte de los pensadores posteriores han desconocido la filosofía de los anteriores. Por ejemplo, la mayor parte de los célebres pensadores modernos y contemporáneos, han desconocido casi por entero la filosofía medieval y en buena medida la clásica griega. Otro, porque habiendo conocido las filosofías precedentes, no han sido capaces de entender sus grandes hallazgos hasta el punto de continuar sus descubrimientos. Así, el tratamiento del conocer natural superior humano, el del intelecto agente, deja mucho que desear a lo largo de la historia de la filosofía, pues salvo raras excepciones está rebajadamente interpretado ${ }^{13}$. El primer motivo es histórico, el segundo de capacidad noética.

En suma, son muchos los niveles cognoscitivos humanos, pero de ordinario las diversas escuelas y sus filósofos se ciñen a alguna vía operativa de la razón. Ahora bien, es claro, por ejemplo, que la razón y la voluntad como potencias, y con más motivo la intimidad humana o acto de ser personal, no pueden ser conocidos por la razón, porque no se pueden abstraer, siendo así que el conocer de esta facultad deriva de la abstracción; en consecuencia, para su conocimiento se requiere de hábitos innatos ${ }^{14}$.

más bien, natos». Polo, L., Antropología trascendental, ed. cit., 357, nota 114.

13 Cfr. nota 2. De los 513 filósofos estudiados en dichas publicaciones, menos de una docena han acertado en la índole del intelecto agente, lo cual equivale a un modesto $2 \%$.

14 «La inteligencia se describe como potencia inmaterial y, por tanto, como no susceptible de recepción. Por eso, la noción de especie impresa no es apropiada: es preferible hablar de ayuda suscitada por la luz del hábito innato del que la inteligencia depende, el cual es inferior al intelecto personal. La sindéresis es un hábito innato que ha de considerarse en orden a la voluntad y a la inteligencia». Polo, L., Antropología trascendental, ed. cit., 184. «La voluntad es una dimensión del alma humana a la que no se puede acceder desde 
Asimismo, no se puede conocer a Dios como ser pluripersonal mirando hacia afuera, sino a través del conocer personal. De manera que los periodos filosóficos de olvido de los hábitos innatos y del acto de ser personal difícilmente darán cuenta de dichos temas, y es claro que tales etapas son mayoritarias en la historia del pensamiento.

Lo que precede indica que «a lo largo de la historia de la filosofía el axioma que L. Polo denomina con la letra 'B' ('La diferencia entre las distintas operaciones $-y$ objetos- es jerárquica') ha sido repetidamente conculcado... La diferencia entre las operaciones intelectuales ha sido advertida con frecuencia de un modo confuso» ${ }^{15}$. La historia de la filosofía admite altibajos, cimas y valles, porque responde a la libertad personal humana. Esto indica que «la historia de la filosofía es el axioma B en conexión con el axioma D ('La inteligencia es operativamente irrestricta merced a los hábitos'), el axioma de los hábitos, y sus varias conculcaciones. En ella se registran avances y retrocesos, así como ciertas divergencias debidas al predominio epocal o de escuela de alguna operación intelectual. Así pues, hay un sentido de la historia exclusivo de la filosofía, cuya contextualización con la cultura es débil. Ésta es una de las razones que explican por qué no ha habido siempre filosofía» ${ }^{16}$, y por qué no necesariamente la habrá.

"A lo largo de la historia de la filosofía o se ha prescindido de ciertos actos o se les ha atribuido una conmensuración con objetos que es incorrecta. La confusión entre operaciones que son distintas no es una verdadera unificación, sino que conculca la axiomática (sobre todo, el axioma de la conmensuración: axioma E ('No hay objeto sin operación') ... La filosofía... deja de ser filosofía en la medida en que prescinde de operaciones intelectuales o las confunde» ${ }^{17}$. La operación preliminar de la razón es la abstracción, cuyo objeto conocido ha sido no solo caballo de batalla de los empirismos y materialismos de todos los tiempos, porque no han logrado dar cuenta de su universalidad, sino también de los racionalismos e idea-

la iluminación de lo sensible, y tampoco parece que reciba especies impresas. Por tanto, la voluntad sólo se puede conocer con un hábito que ha de ser innato». Ibid., 178.

15 Polo, L., Curso de teoría del conocimiento, vol. II, ed. cit., 46.

16 Polo, L., Curso de teoría del conocimiento, vol. II, ed. cit., 228.

17 Polo, L., Curso de teoría del conocimiento, vol. III, en Obras Completas, Serie A, vol. VI, Pamplona: Eunsa, 2015, 20. 
lismos, porque han olvidado la intencionalidad de su objeto conocido ${ }^{18}$, e incluso de algunos realismos, que atribuyen erróneamente la intencionalidad al acto de conocer en vez de al objeto conocido.

Tras esa operación la inteligencia puede proseguir con dos vías operativas divergentes, que son dos declaraciones de insuficiencia de la abstracción: la que declara su escasez respecto de la capacidad cognoscitiva de la inteligencia y la que indica su carencia respecto de la realidad de donde se ha abstraído ${ }^{19}$. La primera -llamada 'vía de abstracción formal' por Tomás de Aquino y 'generalización' y 'negación' por Polo- es inferior a la segunda -denominada 'vía de abstracción total' por el primero y 'razón' por el segundo-. El racionalismo y el idealismo han seguido en buena medida la primera vía, mientras que el realismo clásico ha seguido la segunda ${ }^{20}$. A la

18 Cfr. al respecto: Sanguineti, J.J., Aspectos del conocimiento intencional según Leonardo Polo, en Zorroza, I., Sellés, J.F., (eds.), La teoría del conocimiento de Leonardo Polo, Pamplona: Eunsa, 2018, 15-37; García, J.A., La problemática intencionalidad de a razón humana, o la excesiva superioridad del espíritu sobre lo físico, en Ibid., 233-239; Murillo, J.I., Intencionalidad, causalidad y finalidad, en Ibid., 273- 287.

19 «Siendo la abstracción la operación incoativa, o primera, la inteligencia es capaz de proseguir de dos modos diferentes, que son, en rigor, dos líneas operativas. Dicho de otra manera: desde la abstracción, la inteligencia ejerce dos tipos de operaciones divergentes; por tanto, no hay una prosecución operativa única a partir de la abstracción... Notar que el conocimiento abstractivo no es el más alto equivale a declarar su insuficiencia. Tal insuficiencia es relativa, en primer lugar, a la inteligencia misma y, en segundo lugar, al conocimiento de la realidad; pero estas dos insuficiencias no son iguales; las declaraciones de las insuficiencias de la abstracción son, por tanto, irreductibles (de acuerdo con el axioma de la jerarquía; de aquí que su unificación requiera otra operación)». Polo, L., Curso de teoría del conocimiento, vol. III, ed. cit., 73.

$20 \ll$ El ejercicio del tipo de operaciones cuyos objetos son las ideas generales es predominante en la filosofía moderna y en la actividad científica (piénsese, por ejemplo, en la física-matemática). En la tradición filosófica griega, el pensamiento negativo está menos desarrollado. Ahora bien, sostengo que jerárquicamente la operación de negar es inferior a la razón y que este último tipo de operaciones se ejerce en filosofía, sobre todo, a partir de Platón y de Aristóteles. Parece que si hay una correlación entre la historia de la filosofía y las operaciones cognoscitivas, la negación ha de ser la que primero se ejerce (después de la abstracción). Así ha sido, como hemos visto. Sin embargo, su ejercicio no se desarrolla en los griegos. En cambio, el concepto, el juicio y la búsqueda del fundamento se ejercen con intensidad en la filosofía clásica. A ello se debe, por otra parte, su característica nobleza. Habría que decir, entonces, entre otras cosas, que la filosofía moderna está pensada con operaciones inferiores. Pero, a la vez, el escaso despliegue de la negación en los clásicos les impide distinguirla de la razón con entera nitidez... Los clásicos notaron que la operación de negar no es adecuada para profundizar en lo real, y por eso la ejercieron 
primera vía pertenece la lógica; a la segunda la filosofía de la naturaleza o física clásicamente considerada.

Al margen de la divergencia aludida, hay que indicar que si bien la filosofía moderna ha ejercido la vía generalizante, no ha reparado en los hábitos adquiridos de esta vía. Por su parte, la filosofía realista que ha seguido la vía racional, y que sí ha reparado en los hábitos adquiridos de esta vía, y asimismo en los de la razón práctica, no ha notado que los hábitos adquiridos son más cognoscitivos que los actos u operaciones inmanentes ${ }^{21}$, lo cual es asimismo axiomático, porque los hábitos adquiridos son el conocimiento de sus respectivas operaciones ${ }^{22}$. Por tanto, si en los dos derroteros históricos del ejercicio de las vías operativas de la razón, mínimamente descritas, lo ordinario ha sido ejercerlas reductivamente, con mayor motivo hay que decir que la separación habida entre una y otra ha obligado a olvidar cómo se unen ambas vías. A ésta vinculación Polo la denomina $\operatorname{lógos}^{23}$ y a ella responde la matemática.

En suma, en la serie de la historia de la filosofía primero se ha dado la lógica, luego la física, y en tercer lugar la matemática. Superior a ellas es la

poco, y, en la medida en que lo hicieron, forzaron su alcance. Los modernos la ejercen más y chocan con su limitación». Polo, L., Curso de teoría del conocimiento, vol. III, ed. cit., 83. 21 «Tampoco es claro en la historia de la filosofía que el conocimiento habitual sea el perfeccionamiento de la inteligencia que permite el ejercicio de las operaciones siguientes... Desde luego, no está nada claro en la filosofía moderna, que es 'objetualista' y olvida el conocimiento habitual: no admite que el hábito sea un acto cognoscitivo, y además superior al conocimiento objetivo (por eso, la advertencia de la insuficiencia del conocimiento objetivo da lugar al irracionalismo). La teoría del conocimiento clásica admite los hábitos intelectuales, pero no está muy segura de que el conocimiento habitual sea un conocer en acto, sino que lo interpreta como potencial al compararlo con el conocimiento en acto que, en sentido estricto, sería el operativo. Correlativamente, los hábitos no se interpretan como preparaciones para operaciones desde su superioridad sobre la operación a la que siguen, sino que, más bien, se enfoca el conocimiento habitual como una conservación del conocimiento operativo». Polo, L., Curso de teoría del conocimiento, vol. III, ed. cit., 38. 22 Cfr. al respecto: Collado, S., Noción de hábito en la teoría del conocimiento de Polo, Pamplona, Eunsa, 2000; Ferrer, U., Los hábitos cognoscitivos: del saber hablar al conocer por principios, en Zorroza, I., Sellés, J.F., (eds.), La teoría del conocimiento de Leonardo Polo, Pamplona, Eunsa, 2018, 241-254.

23 «He propuesto llamar logos a la unificación operativa. El estudio del logos sólo es posible si las confusiones aludidas, que tan frecuentes son en la historia de la filosofía, se resuelven». Ibid., 62. Cfr. al respecto: Falgueras, I., Logos y legein, en la teoría del conocimiento de Leonardo Polo, en Zorroza, I., Sellés, J.F., (eds.), La teoría del conocimiento de Leonardo Polo, Pamplona: Eunsa, 2018, 83-131. 
ética, que es posibilitada por el hábito innato de la sindéresis, superior a los actos y hábitos adquiridos de la razón en sus diversas vías y sus unificaciones. Pero previa a la ética son la psicología y la sociología, porque ambas estudian lo natural humano -facultades y manifestaciones- sin considerar su perfeccionamiento ético. Superior a la ética es la metafísica, porque su tema, a distinción de aquélla, no es el obrar sino el ser, en concreto, los actos de ser reales extramentales. Y superior a la metafísica es la antropología trascendental, porque superior los seres no personales es el propio ser personal, pues lo libre es superior a lo necesario.

\section{I.2. LA FILOSOfía PROGRESA EN LA MEDIDA EN QUE SE DESCUBREN SUPE- RIORES ACTOS DE SER}

Aristóteles descubrió que Dios es acto, pero admitió que toda realidad al margen del Acto puro es potencial. «El incremento temático de la filosofía es la obra de los grandes filósofos. No merecen ese calificativo dos tipos de filósofos; en primer lugar los transmisores de lo logrado por otros... En segundo lugar, los que advierten dificultades en lo logrado sin añadir nada» ${ }^{24}$. Tomás de Aquino superó a Aristóteles porque descubrió la distinción real 'essentia-actus essendi en lo creado. Por tanto, advirtió que en lo no divino (y no sólo en el conocer), también se dan actos sin potencia, a saber, los actos de ser. Polo superó al Aquinate porque distinguió entre distintos actos de ser creados -el personal o ser-con y el no personal o puramente persistente-, y asimismo entre sus respectivas esencias ${ }^{25}$. En el personal descubrió, además, que es libre, cognoscente y amante, solucionando así por elevación las inquietudes de la antropología moderna.

\section{Se PUeden JeRARQuizar los FILÓsofos y SUS ESCUELAS DESDE VARIAS DISCIPLINAS FILOSÓFICAS}

Recuérdese que en el libro introductorio de su Metafísica Aristóteles

24 Polo, L., Introducción a la filosofía, en Obras Completas, vol. XII, Pamplona, Eunsa, 2015, 179. Ejemplo de ese tipo de filósofos son, por ejemplo, los cometadores tomistas que tras el descubrimiento tomista de la distinción real acto de ser-esencia han tratado de él, pero lejos superar la mente del Aquinate cabe dudar si están a su altura.

25 «No se trata de ir más allá de la distinción real de ser y esencia, sino de entenderla mejor, ampliándola - sin suponerla- al ser personal humano (y ascendiendo desde él al Misterio del ser personal divino)». Polo, L., Antropología trascendental, ed. cit., 166. 
puso orden jerárquico a los filósofos clásicos griegos precedentes a él y lo hizo desde su Física ${ }^{26}$, no desde la Metafísica, porque ésta es el saber que él inaugura precisamente en ese libro y que no deja terminado, porque -según él- la metafísica es 'la ciencia que se busca'. Dado que las causas de la realidad física son cuatro, siendo la material inferior a la formal, ésta a la eficiente, y ésta a la final, es tesis aristotélica que los pensadores presocráticos se distinguen entre sí según jerarquía de acuerdo con la preeminencia que otorgaron a una u otra de tales causas. Ahora bien, como la Física filosófica no tiene como tema el acto, sino lo potencial, porque la realidad física es siempre de esta condición, aunque en ella no todo esté en el mismo plano ni valga lo mismo, dado que la Física no juega con lo que es perfecto, no se puede axiomatizar. Por tanto, ofrecerá una jerarquía entre los diversos filósofos menos aguda que la que ofrezcan las disciplinas filosóficas que son axiomatizables.

En cambio, Polo pone orden jerárquico a los filósofos precedentes desde las disciplinas filosóficas que se pueden axiomatizar, porque sus temas son actos perfectos. Éstas, de inferior a superior, son las siguientes:

\section{II.1. LA TEORÍA DEL CONOCIMIENTO ${ }^{27}$}

cuyos temas, los objetos pensados, lo son por los 'actos u operaciones inmanentes' de la razón y cuyo método es doble: por una parte, los 'hábitos adquiridos' de la razón teórica, que permiten conocer los actos y, por otra, una vertiente del 'hábito innato de la sindéresis', que permite conocer los hábitos adquiridos de la razón.

\section{II.2. LA ÉTICA}

cuyos temas son -además de los bienes reales extramentales- los 'actos y virtudes' de la voluntad y cuyo método es doble: por una parte, los 'actos

26 «Al escribir su pequeño compendio de historia de la filosofía, justamente al empezar la Metafísica es donde aparecen las causas: la manera que tiene Aristóteles de ordenar a los pensadores anteriores a él». Polo, L., El conocimiento del universo físico, ed. cit., 113. Cfr. asimismo: La esencia del hombre, en Obras Completas, Serie A, vol. XXIII, Pamplona: Eunsa, 2015, 117.

$27 \mathrm{Al}$ respecto ha escrito Falgueras: «La exposición (de la teoría del conocimiento de Polo) remite de modo asiduo, para hacerse más asequible, a los momentos de la historia del pensamiento humano en que han ido siendo ejercidos y establecidos (los objetos con sus operaciones y hábitos). Nunca antes se ha ofrecido una comprensión más intensa de 
de la razón práctica', que conocen los bienes reales mediales, y sus 'hábitos' que conocen tales actos, y por otra, una vertiente distinta del 'hábito innato de la sindéresis', que conoce los actos y virtudes de la voluntad y el bien último al que ésta se abre.

\section{II.3. LA METAFÍSICA}

cuyos temas son los 'actos de ser reales extramentales' y cuyo método noético es el 'hábito innato de los primeros principios'.

\section{II.4. LA ANTROPOLOGÍA TRASCENDENTAL}

cuyos temas son los 'co-actos de ser' o trascendentales personales, y cuyo método noético es el 'hábito de sabiduría'.

Explicitemos brevemente, según Polo, estos ordenamientos:

1) Desde la axiomatización de la teoría del conocimiento Polo afirma «que toda teoría del conocimiento humano que no reconozca los axiomas, o que se haga al margen de los axiomas, es falsa... La teoría del conocimiento de Kant, de Hegel, o de cualquiera, si no reconocen los axiomas, son equivocaciones. Pues sí, lo son: la axiomática lo implica... Quien haga una teoría del conocimiento humano al margen de la axiomática, negándola, no ateniéndose a ella, se equivoca... Si es una axiomática en el sentido propio de la palabra, determina la verdad de la teoría del conocimiento, la única posible teoría del conocimiento humano en términos de corrección... El que niegue algún axioma está estudiando el conocimiento en el vacío» ${ }^{28}$.

Más aún, como los axiomas están vinculados entre sí, quien conculque uno, en rigor, conculca los demás. Nótese que en teoría del conocimiento rigen las dualidades: por una parte, la conformada entre objetos pensados y operaciones inmanentes; por otro, la que se da entre éstas y sus hábitos adquiridos respectivos; y otra, la que se da entre los hábitos adquiridos y el

las filosofías habidas históricamente que la que se ofrece en estos volúmenes (I-IV del Curso de teoría del conocimiento de Polo). La historia de la filosofía cobra todo su vigor y sentido cuando se acredita en ella el progreso de las operaciones y de los hábitos intelectuales humanos, con lo que se evitan adhesiones acríticas, se señalan las confusiones y se respetan los hallazgos». Falgueras, I., Introducción general a las Obras Completas de Leonardo Polo, en Polo, L., Evidencia y realidad en Descartes, en Obras Completas, vol. I, ed. cit., 79. 28 Polo, L., Curso de teoría del conocimiento, vol. I, ed. cit., 36. 
hábito innato que los posibilita: una dimensión de la sindéresis. Pues bien, en la teoría del conocimiento de Polo se dice qué autores se ajustan a los axiomas y cuales no y por qué.

2) Por lo que respecta a la ética, Polo escribe, en una primera aproximación, que «en la historia de la filosofía se han dado, sobre todo, tres tipos diversos de ética: $1^{\text {a }}$ ) Ética de bienes: que considera como único fin la consecución de bienes, especialmente materiales. No es una ética suficiente, pues los bienes materiales son necesarios, pero insuficientes por sí mismos. $2^{a}$ ) Ética de normas: es la ética kantiana. Se basa en el cumplimiento del deber. También es insuficiente, porque el deber es un medio para expresar o conseguir el amor. De lo contrario, es una ética que no es moral, sino moralista. El deber por el deber mismo es frío. $3^{a}$ ) Ética de virtudes: se trata de centrar el actuar humano libre en el amor, pues esto es lo que personaliza y perfecciona realmente al hombre. Aquí el tema central es el enderezamiento de la libertad. La tesis fundamental para basar la ética es la siguiente: 'Una ética verdadera debe tener en cuenta los tres elementos: bienes, normas y virtudes' $\rangle^{29}$.

Lo que precede indica cuatro cosas: $1^{\mathrm{a}}$ ) Que la ética de sólo bienes suele ponerse al margen del bien último, el cual es irrestricto, inmaterial y susceptible de saciar a la voluntad humana. Pero como los bienes mediales no son acto, sino potenciales, esta ética es inferior a la que se ciñe a las normas, las cuales son actos de la razón práctica. $2^{\mathrm{a}}$ ) Indica también que la ética de solo normas se deja en el tintero no sólo los bienes reales mediales y el último, sino también los hábitos de la razón práctica, y sobre todo, las virtudes de la voluntad, y asimismo la norma de la sindéresis, la cual impele a actuar. Ahora bien, como las normas de la razón práctica son inferiores a las virtudes, la ética normativa es inferior a la de virtudes. $3^{a}$ ) Indica asimismo que la ética de solo virtudes, al tomar a éstas como fin, conculca la intencionalidad de alteridad de la voluntad y, por tanto, su correspondencia con los bienes reales mediales y sobre todo con el último, a la par que omite las normas de la razón práctica y la norma básica de la sindéresis. $4^{\mathrm{a}}$ ) Y, desde luego, toda ética que no se funde en la sindéresis carece de fundamentación, pues sólo ésta permite conocer el bien último, a la par que activar a la razón práctica con actos y hábitos, y a la voluntad

29 Polo, L., Filosofía y economía, en Obras Completas, Serie A, vol. XXV, Pamplona: Eunsa, 2015, 423. 
con actos y virtudes en correspondencia con los bienes.

Por eso, en una primera aproximación, hay que decir que la ética requiere de esas tres bases. Pero éstas se pueden reducir a dos, a saber, bienes y normas, puesto que, a distinción de los bienes externos, las virtudes son bienes internos, lo cual Polo también advirtió: «la ética es dual, pues trata de las normas y de los bienes. A su vez, las normas morales ofrecen una dualidad: el primer principio moral (que se encierra en la sindéresis, hábito innato que... también es dual) y las leyes directamente vigentes que, a su vez, son positivas o negativas. Por su parte, los bienes son internos (virtudes) e intentados o externos, según la dualidad de medios y fines $»^{30}$.

Nótese, pues, que la ética está conformada por una pluralidad de dualidades ${ }^{31}: 1^{\circ}$ ) La de bienes y normas. $2^{\circ}$ ) Los bienes pueden ser externos o internos. A su vez, los bienes externos son mediales o último, y los internos son actos o virtudes de la voluntad. $3^{\circ}$ ) Las normas son, o bien de la razón práctica, o bien de la sindéresis. En todo caso se ve la jerarquía entre los dos miembros de las dualidades, pues los bienes mediales son inferiores a las normas de la razón práctica; éstos a las virtudes, éstas a la sindéresis y ésta al bien último. Según esta jerarquía, que es axiomática, se pueden clasificar los diversos filósofos que han ofrecido propuestas en ética.

3) Por lo que respecta a la metafísica, «los primeros principios son los axiomas de la metafísica» ${ }^{32}$, y estos son el acto de ser del universo, el acto de ser divino y la dependencia del primero respecto del segundo (su dualidad parece neta). Tales principios han sido muy poco advertidos en la historia de la filosofía. Para Polo es claro que Tomás de Aquino los advirtió, pues de lo contrario no hubiese escrito aquello de «licet Causa Prima, quae Deus est, non intret in essentiam rerum creaturarum; tamen esse, quod rebus creatis inest, non potest intelligi nisi ut deductum ab esse divinor ${ }^{33}$. Pero obviamente no lo han advertido los racionalistas y empiristas de todas las épocas. Tampoco

30 Polo, L., Antropología trascendental, ed. cit., 165.

31 Cfr. al respecto mi trabajo: The Antropological Foundation of Ethics and its Dualities, en Journal of Polian Studies, 1 (2014) 47-77.

32 Polo, L., Curso de teoría del conocimiento, II, ed. cit., 262. Ibid., III, ed. cit., 61 y 75. Ibid., IV, en Obras Completas, Serie A, vol. VII, Pamplona: Eunsa, 2019, 650, nota 131. Antropología trascendental, ed. cit., 243, nota 4. El conocimiento del universo físico, ed. cit., 188.

33 Tomás de Aquino, De Potentia, q. 3, a. 5, ad 1. Cfr. Polo, L., El Ser I, en Obras Completas, Serie A, vol. III, Pamplona: Eunsa, 2015, 15. 
quien quiso restablecer la ontología en el s. XX: Heidegger ${ }^{34}$. De modo que la axiomática de la metafísica se muestra mucho más exigente que la de la teoría del conocimiento y la de ética para ordenar según jerarquía las diversas propuestas de los filósofos.

A la par, Polo sostuvo que «la cuestión del orden de los trascendentales (metafísicos) proporciona el mejor criterio para entender la historia de la filosofía. Ello comporta que es inevitable inquirir por el primer trascendental. El idealismo sostiene que el primer trascendental es la verdad. Para el nominalismo el primer trascendental sería el bien. Para el realismo el primer trascendental es el ser. Pero además de servir para caracterizar a fondo las distintas posturas filosóficas, el planteamiento de la cuestión del primer trascendental, proporciona el criterio para averiguar cual de esas filosofías es la verdadera. Este criterio reside en lo siguiente: el orden de los trascendentales debe permitir su convertibilidad, es decir, seguir entendiendo a los otros trascendentales como tales. Con otras palabras, si al aceptar un trascendental como primero y los otros no pueden entenderse como tales, esa filosofía es equivocada; es decir, no permitir la convertibilidad equivale a excluir el carácter trascendental de los que no son el primero» ${ }^{35}$. Según

34 «Lo que llama Heidegger diferencia ontológica no debe confundirse con la distinción real de essentia y esse». Polo, L., Nominalismo, idealismo y realismo, ed. cit., 24.

35 Ibid.,157. A eso añade que «la discusión acerca del primer trascendental permite caracterizar las grandes variaciones de la historia de la filosofía. ¿Qué tipos de filosofía hay? En definitiva, el idealismo y el nominalismo, el realismo, y el monismo. En nuestros días, está en alza el pulcrismo, es decir, la tesis de que la belleza es lo primero, por tanto, la filosofía se entiende como filosofía del arte... El realismo se describe como aquella postura metafísica que formula la tesis de que el primer trascendental es el ser... El idealismo es aquella filosofía que promulga la prioridad, o autofundamentación, de la verdad. El nominalismo es aquel planteamiento filosófico que prima el bien. Para el monismo el primer trascendental es el uno... Es inevitable preguntar quién tiene razón al determinar cuál es el primer trascendental. El único argu $\neg$ mento pertinente para responder a esa pregunta reside en que el orden ha de ser compatible con la conversión. Por tanto, será correcta aquella postura acerca de la prioridad que permita admitir que los otros trascendentales, aunque no sean el primero, también son trascendentales. Si al elegir un trascendental como primero, los otros no se pueden convertir con él, es decir, si dejan de ser trascendentales, el planteamiento no es correcto. El idealismo es incorrecto sobre todo porque no puede garantizar que el bien sea trascendental; no puede convertir la verdad con el bien. El voluntarismo no puede convertir el bien con la verdad ni tampoco con el ser. Para Plotino, ni la verdad ni el ente, que son la segunda hipóstasis, son trascendentales, porque lo único trascendental es el uno. De manera que ni la verdad, ni el bien 
esta exigencia el realismo es superior al idealismo y al nominalismo.

4) En cuanto a la antropología trascendental, aunque se pueden encontrar intuiciones certeras al menos en el $2^{\circ}$ Scheler ${ }^{36}$ y en Ratzinger (y desvaídos destellos de ellas en Nédoncelle ${ }^{37}$ y V. Frankl ${ }^{38}$-seguramente hay más autores que hayan tenido intuiciones semejantes-), cabe decir que, en rigor, es un hallazgo poliano de primera magnitud. Por tanto, la jerarquización entre los filósofos habidos que ofrece esta disciplina filosófica axiomática es muy estricta. En efecto, como Polo descubrió por encima de los trascendentales metafísicos los antropológicos, y dado que éstos son jerárquicos, cabe hacer un balance de la historia de la filosofía desde ellos más radical que desde los metafísicos. Así, quienes hayan primado al amor sobre el conocer serán filósofos superiores ${ }^{39}$, y éstos lo serán respecto de quienes hayan primado la libertad ${ }^{40}$, y éstos sobre quienes priman la coexistencia ${ }^{41}$.

son el primer trascendental... El ser puede ser el primer trascendental siempre y cuando la prioridad del ser permita la conversión, es decir, seguir diciendo que la verdad, el bien, y el pulchrum son trascendentales; de lo contrario, no. Hay algunos tipos de realismo que no permiten la conversión y, por tanto, son falsos; en rigor, más falsos que el idealismo, porque esa prioridad del ser que no permite la conversión implica que tampoco el ser se entiende como trascendental. Este es el caso del materialismo». Ibid., 194-196. Cfr. también: Antropología trascendental, ed. cit., 88; Persona y libertad, en Obras Completas, Serie A, vol. XIX, Pamplona: Eunsa, 2017, 243-244.

36 Cfr. mi trabajo: Intuición y perplejidad en la antropología de Scheler. Introducción, textos, glosas. Cuadernos de Anuario Filosófico, Serie Universitaria, nº 216, Pamplona: Servicio de Publicaciones de la Universidad de Navarra, 2009.

37 Cfr. mi trabajo: La antropología trascendental de Maurice Nédoncelle, Madrid: Ápeiron, 2015. 38 Cfr. mi trabajo: ¿Es trascendental la antropología de Viktor E. Frankl?, Madrid: Ápeiron, 2016.

39 «Los trascendentales personales, son: el ser personal... el trascendental en correspondencia no simétrica con la verdad, que es el conocer, y el trascendental que se corresponde con el bien sin simetrizarlo, que es el amor... Pues bien, la libertad es, como acto de ser, el primer trascendental personal, según el cual vige la conversión de los trascendentales que acabo de enumerar. La persona no es si no es libre; el intellectus ut actus, la luz en su separación pura, otorgadora de inteligibilidad, también es libre; el amor sólo es donal si es libre». Polo, L., Persona y libertad, ed. cit., 247.

40 Cfr. Ibid., 177.

41 «La comprensión de la libertad y la manera como se propugna o como se intenta practicar, el alcance que se le concede, depende de cómo se entienda lo radical en el hombre, y esto nos lleva directamente a la historia del pensamiento humano, más incluso que a la historia de la filosofía, porque aquí interviene también la teología y otras dimensiones culturales». Ibid., 178. 
Además de las precedentes disciplinas, que son susceptibles de axiomatización, Polo ha puesto las bases de la axiomatización de otras dos superiores a las precedentes: la de la teología natural desde el conocer personal, y la teología sobrenatural llevada a cabo con la elevación sobrenatural de éste mediante la fe.

En cuanto a la primera, la teología natural desde el conocer personal, cabe decir que sus puntos fuertes son éstos: $1^{\circ}$ ) Como el conocer humano a nivel de ser está personalmente abierto a su tema y no se entiende sin él, también éste es personal. $2^{\circ}$ ) Como dicho conocer y su tema son personales, y ningún conocer personal puede ser sin tema personal, esto indica que su tema, por personal, es pluripersonal. $3^{\circ}$ ) Como el conocer personal humano está transido de libertad y amor, también el ser pluripersonal divino es libre y amante. $4^{\circ}$ ) Como el amar personal, al que está unido el conocer personal requiere tres dimensiones -aceptar, dar y don- cada una de ellas debe ser semejante a cada una las personas divinas. Con esto se puede dirimir la jerarquía entre las teologías naturales existentes, y asimismo entre las religiones naturales habidas en la historia.

En cuanto a la segunda, a la teología sobrenatural, Polo indica que las exigencias de la credendidad son éstas: $1^{\text {a) }}$ "Una verdadera iniciativa de la ultimidad debe estar por completo fuera del alcance de nuestra capacidad de averiguación. $2^{\text {a) }}$ Lo revelado ha de ser compatible con todo lo que nuestra inteligencia pueda descubrir como verdadero por sí misma. $3^{\text {a) }}$ Para que una revelación sea admisible, se requiere la congruencia del revelante consigo mismo. Si el revelante es Dios, tanto el fin como el modo de la revelación han de estar por encima de lo que llamaríamos cierta necesidad del revelante, pues si en algún sentido necesita revelársenos, no es superior a nosotros. $4^{\mathrm{a}}$ ) Una condición de la revelación auténtica es que ha de ser congruente tanto con el revelante como con quien la recibe. La verdadera revelación nos ha de hacer mejores. Si la revelación envileciera al hombre no sería verdadera. Tampoco si deja igual al hombre. Asimismo, la congruencia de la revelación requiere que lo revelado afecte a la totalidad de la persona. Una revelación que afecte sólo a una dimensión humana no es congruente» ${ }^{42}$ con la índole del ser humano. Con esto se puede dirimir

42 Polo, L., La originalidad de la concepción cristiana de la existencia, en Obras Completas, Serie A, vol. XIII, Pamplona: Eunsa, 2015, 75-76. 
la jerarquía entre las teologías sobrenaturales existentes, y asimismo entre las religiones que se tienen a sí mismas por sobrenaturales.

III. ¿Cómo progresar tras L. Polo?

Si los actos de conocer y los actos de ser no son irrestrictos, ¿la filosofía puede progresar irrestrictamente? Formulado de otra manera: si es verdad que Polo es el que más descubrimientos ha realizado en los actos de conocer y en los actos de ser, ¿cómo superar lo por él descubierto? Él decía que «la historia de la filosofía enseña mucho; también que la razón puede con la historia. Pero no es cierto que ese dominio sobre la historia se consume de tal modo que lo que venga después sea un puro comentario a lo ya descubierto, o que ya no se pueda descubrir nada más. Ni estupor ni consagración definitiva» ${ }^{43}$. También indicaba que "estamos actualmente en una fase de falta de renovación filosófica. No hay que extrañarse, porque los momentos estelares de la filosofía no son abundantes, y tampoco duran mucho ${ }^{44}$. Durante siglos enteros la filosofía está en baja; se mantiene, se cultiva, en el sentido de una simple transmisión de lo adquirido, pero no hay creatividad. Hoy estamos en una de esas fases ${ }^{45}$.

Si la filosofía de Polo obedece a un punto álgido de la historia de la filosofía, ¿cómo avanzar sobre ella? Primero, haciéndose cargo de ella, pues «un filósofo puede ser tan contemporáneo como cualquiera, porque si lo que pensó es verdad, sigue siendo verdad. Lo que interesa es saber cómo lo pensó, cómo llegó hasta ahí; si se puede dar un paso más, serle fiel a ese pensador. La lealtad a un gran pensador no consiste en repetirle, sino en tratar de entenderle bien y, luego, intentar avanzar, ir un poco más allá sin petulancias... La filosofía siempre es capaz de ir adelante, siempre se puede saber más. En la medida en que se deshace la rigidez de la tradición (que sobre todo está en los libros), en la medida en que está viva en uno, no paraliza, sino que impulsa... Admitir que no queda nada por averiguar (o remitirse a la otra vida) sería contradictorio: la filosofía ha de reemprender la marcha» ${ }^{46}$.

43 Polo, L., Introducción a la filosofía, ed. cit., 41.

44 Cfr. al respecto: Polo, L., Presente y futuro del hombre, en Obras Completas, Serie A, vol. X, Pamplona, Eunsa, 2015, 232-236; Epistemología, creación y divinidad, ed. cit., 52. Cfr. Yepes, R., Leonardo Polo y la historia de la filosofía, en Anuario Filosófico, XXV/1 (1992) 101124.

45 Polo, L., Introducción a la filosofía, ed. cit., 41.

46 Ibid., 42. 
Pero hacerse cargo de la filosofía poliana no es tarea fácil, ni rápida. Lo primero, por la profundidad; lo segundo, porque implica hacerse cargo de la filosofía de los mejores pensadores de la historia de la filosofía, pues a distinción de los modernos y contemporáneos, Polo ha conocido las piezas magistrales de los filósofos más relevantes a lo largo de todos los tiempos, las ha tenido en cuenta desde una altura inusitada ${ }^{47}$, y ha ido más allá de ellas.

Superar a Polo podría significar ejercer actos de conocer superiores a los por él ejercidos y advertir sus respectivos temas, pero si los actos de conocer humanos son los que son y Polo ha ejercido los superiores y con ellos ha descubierto sus temas, ¿cómo superarle? Esto es, sin duda, aporético. Pero para Polo «lo aporético se condensa en lo inacabado de la obra completa de los grandes filósofos. Los aciertos, los logros importantes -y más cuanto más importantes-, dejan abierto lo que ha de colmarse: lo no dicho, lo no pensado todavía de lo que ha sido pensado. Eso es justamente lo que los filósofos transmisores no ven y lo que despierta la perplejidad complementaria de los filósofos testigos» ${ }^{48}$.

Para superar a Polo cabría alegar dos cosas: una, que cabe ejercer más que Polo el conocer superior -el personal-, si éste es elevado por Dios mediante la fe sobrenatural, pero esto es asimismo aporético por doble motivo: porque el conocer propio de la fe no es filosofía sino teología sobrenatural, y porque Polo también lo ejerció. Otra, encontrar temas de envergadura que dejó Polo sin investigar. Pero, como hemos visto, Polo ha axiomatizado las disciplinas filosóficas más relevantes, además de perfilar mejor que la tradición precedente otras como la física filosófica, la biología, el carácter distintivo de la corporeidad humana, la lógica, la matemática, la psicología, la sociología, la historia, la teoría de la empresa y la economía,

47 «D. Leonardo no ecluyó de su filosofar a ningún filósofo, siempre supo encontrar en todos algo de verdad, y en un sentido fiel al que cada uno de ellos habría propuesto». Falgueras, I., Leonardo Polo, investigador de la verdad, en Leonardo Polo 1926-2013, In Memoriam, Pamplona: Servicio de Publicaciones de la Universidad de Navarra, 2013, 43. «Polo... es un gran conocedor de la historia del pensamiento... Además de los libros expresamente dedicados a la historia del pensamiento, está la multitud de referencias a filósofos con que Polo enriquece sus obras más temáticas». García González, J.A., Prólogo a Polo, L., Estudios de filosofía moderna y contemporánea, en Obras Completas, vol. XXIV, Pamplona: Eunsa, 2015, 11-12.

48 Polo, L., Introducción a la filosofía, ed. cit., 180. 
el estatuto del lenguaje y de la cultura, la teoría de la educación... ¿Qué queda por hacer? De seguro que se puede profundizar más en cada una de ellas, pero hay otros temas no suficientemente tratados por Polo que son relevantes: por ejemplo, la familia, la estética, la afectividad. Ahora bien, es claro que tales temas no constituyen nuevos actos de ser, sino matices en los ya descubiertos.

La disciplina filosófica superior y en la que más descubrimientos ha ofrecido Polo es la antropología trascendental. Y hay al menos un tema nuclear que Polo dejó sin responder: ¿Por qué contamos desde el momento de la concepción con los niveles superiores del conocer humano -los hábitos innatos y el conocer personal- y no somos conscientes de ellos desde ese momento? Una posible respuesta es que la conciencia no es el conocer humano superior ${ }^{49}$. Otra, que el conocer superior en nosotros va de más a menos a lo largo del tiempo, pues omitimos el ejercicio del superior en la medida en que nos obcecamos por ejercer más el inferior. Dicho de otro modo: en la medida en que nos preocupamos de la esencia del hombre (yo, voluntad e inteligencia) y de la naturaleza corpórea humana desde la sindéresis, más nos olvidamos de nuestro acto de ser personal (que remite personalmente a Dios), es decir, menos ejercemos el hábito de sabiduría y el

49 Hay muchos niveles de conciencia. La inferior es la sensible, que es el conocimiento de los actos de conocer de los sentidos externos en la medida en que éstos se ejercen. A tal conciencia se llama 'sensorio común' o 'percepción sensible'. A nivel de inteligencia se da la llamada 'conciencia concomitante', que no es otra cosa que el hábito adquirido que es inseparable de la operación de la inteligencia a la que conoce, la cual presenta, tiene, el objeto conocido; en rigor, tal conciencia equivale a 'conozco que pienso algo'. Esta conciencia es distinta de la llamada 'conciencia moral', la cual no se reduce a los actos de la razón práctica, los cuales son rectificables y concretan el impulso a actuar propio del hábito innato de la sindéresis, pues la conciencia moral está más vinculada con los hábitos de la razón práctica. Por otra parte, la sindéresis es la conciencia del yo, de la personalidad; con ella somos conscientes de nuestras potencias y de su estado. Es la conciencia del disponer humano. Pero la conciencia del yo no es la conciencia de sí como persona, es decir, del ser personal que uno es, sino inferior a ella. ¿Somos conscientes de nuestro ser? Sí, porque somos conscientes de que éste es filial. Pero esta conciencia es inferior al ser que somos. En efecto, las conciencias arriba indicadas son conoceres superiores al tema que conocen. En cambio, el conocer que alcanza nuestro ser es inferior a éste, y asimismo el conocer personal humano que busca al Dios pluripersonal es inferior a él. Por tanto, siempre sabemos poco el ser que somos y el ser que Dios es. Además, si con el transcurso de la vida se debilita libre y culpablemente la filiación, más se debilita la conciencia de la filiación, porque ésta es inferior y dependiente de aquélla. 
conocer personal; a la par, en la medida que más nos ocupamos de algunos aspectos de la esencia del mundo con nuestra razón, más nos olvidamos de su acto de ser (que remite impersonalmente a Dios), o sea, menos ejercemos el hábito de los primeros principios.

En rigor, lo que precede recuerda al olvido de siempre: intentar reconocernos en nuestra esencia es correlativo del olvido de los actos de ser, en primer lugar del divino, y del de las demás personas creadas; en segundo lugar, del propio; y en tercer lugar, del acto de ser del universo físico. ¿Por qué nos olvidamos de los actos de ser al obcecarnos en nuestra esencia? Porque ésta es posesiva y el acto de ser no se puede poseer. Además, como la esencia del hombre está vinculada a la naturaleza corpórea humana y dado que el carácter distintivo de ésta es el 'tener', ceñirse a ella acarrea el olvido del ser. El acto de ser humano no es 'poseedor' sino 'donante', 'oferente', y por encima de eso, 'aceptante'. Lo radicalmente distinto de 'poseer' no es 'carecer' (pues por ser negativo no se distingue realmente de poseer, sino solo lógicamente). Lo radicalmente distinto de 'poseer' es 'desbordar', y la persona humana desborda en la medida en que 'acepta' a Dios ${ }^{50}$.

Bien mirado, lo que precede implica un salto, o si se quiere, un abandono de un límite que es superior al mental -el del conocimiento objetivo, detectado y abandonado por Polo-. Al igual que en el abandono del límite mental se parte de él, en éste no se trata de prescindir de la esencia del hombre, asunto imposible, sino de no estar limitados a ella. De otra manera: para alcanzarse a conocer como personas hay que abandonar la pretensión de identidad: no querer reconocerse como quien se es en la esencia del hombre. Al abandonar la limitación que ofrece la esencia del hombre nos abrimos por el hábito de sabiduría al acto de ser personal, y por éste al Dios pluripersonal, solo desde el cual cabe un conocimiento completo del acto de ser personal propio. Así como detectar que el conocimiento objetivo es límite mental depende de los hábitos adquiridos ${ }^{51}$, así detectar que la esen-

50 Esta tesis tiene un marcado paralelismo con esta afirmación: «el que quiera salvar su vida la perderá; pero el que ha perdido su vida por mí, la encontrará». Mt., XVI, 25. Como es claro, esto implica la distinción real entre la vida y el viviente, o si se quiere, entre la esencia y el acto de ser.

51 «La interpretación que propongo de la héxis aristotélica debe ser entendida como un recurso frente a la limitación de la presencia mental en el hombre caído, que suple la profundidad del conocimiento presencial de Adán». Polo, L., Epistemología, creación y divinidad, ed. cit., 52-53. 
cia del hombre es límite depende de los hábitos innatos superiores a ella ${ }^{52}$.

Conclusiones

Las podemos resumir en las siguientes:

1a) Si se admite que los niveles del conocer natural humano son plurales y se distinguen entre sí según una neta jerarquía, los autores y las corrientes de pensamiento que hayan ejercido los superiores serán de altura noética superior a quienes se hayan ajustado más a los inferiores. Polo ha descubierto y perfilado mejor que los precedentes filósofos de la historia los diversos niveles cognoscitivos humanos y ha dado más relevancia a los superiores que a los inferiores. Por tanto, se puede decir que está en la cúspide del conocer humano.

2a) Si se admite que los distintos actos de lo real se distinguen entre sí según una neta jerarquía, los autores y las corrientes de pensamiento que hayan descubierto los superiores serán de mayor altura temática que los que se hayan ajustado a temas potenciales o a actos reales inferiores. Polo ha descubirto y perfilado mejor que los precedentes filósofos de la historia los diversos actos de ser reales y ha dado más relevancia a los superiores que a los inferiores. En consecuencia, se puede decir que está en la cúspide de los temas reales.

$3^{a}$ ) Si se admite que se pueden jerarquizar los filósofos y sus diversas corrientes de pensamiento desde las diversas disciplinas filosóficas, en especial las que son axiomatizables, porque sus temas son actos sin potencia -al menos, la teoría del conocimiento, la ética, la metafísica y la antropología trascendental-, entonces Polo, que ha llevado a cabo esta jerarquización, está en la cúspide de ella.

Si las tres tesis precedentes son correctas, se abre el problema de cómo proseguir la filosofía tras Polo, pues es manifiesto que siempre se pueden ejercer mejor los diversos actos de conocer y, consecuentemente, descubrir los temas reales con más profundidad. Si la propuesta metódica de Polo del abandono del límite mental ha permitido conocer mejor los actos de ser y las esencias reales, y ese método coincide con el ejercicio de los hábitos

52 El límite mental obedece al pecado original, porque marca una distancia no debida entre la naturaleza corpórea del hombre y la inteligencia. El límite que ofrece la esencia del hombre es debida asimismo al pecado original, porque la esencia del hombre está directamente vinculada a la naturaleza corpórea humana, y como ésta está lesionada debido al pecado de origen, la esencia está en exceso inclinada a aquélla para paliar su insuficiencia. A esto obedece seguramente la expresión 'proni ad peccatum'. 
adquiridos y de los innatos, por encima de ellos está el conocer personal, del cual tales hábitos dependen. Por tanto, a mejor ejercicio del conocer personal, mejor filosofía.

\section{REFERENCIAS BIBLIOGRÁFICAS.}

Collado, S., Noción de hábito en la teoría del conocimiento de Polo, Pamplona: Eunsa, 2000.

Falgueras, I., Introducción general a las Obras Completas de Leonardo Polo, en Polo, L., Evidencia y realidad en Descartes, en Obras Completas, Serie A, vol. I, Pamplona: Eunsa, 2015.

Falgueras, I., Leonardo Polo, investigador de la verdad, en Leonardo Polo 1926-2013, In Memoriam, Pamplona: Servicio de Publicaciones de la Universidad de Navarra, 2013.

Falgueras, I., Logos y legein, en la teoría del conocimiento de Leonardo Polo, en Zorroza, I., Sellés, J.F., (eds.), La teoría del conocimiento de Leonardo Polo, Pamplona: Eunsa, 2018, 83-131.

Ferrer, U., Los hábitos cognoscitivos: del saber hablar al conocer por principios, en Zorroza, I., Sellés, J.F., (eds.), La teoría del conocimiento de Leonardo Polo, Pamplona: Eunsa, 2018, 241-254.

García González, J.A., Prólogo a Polo, L., Estudios de filosofía moderna y contemporánea, en Obras Completas, Serie A, vol. XXIV, Pamplona: Eunsa, 2015, 11-12.

García, J.A., La problemática intencionalidad de la razón humana, o la excesiva superioridad del espíritu sobre lo físico, en Zorroza, I., Sellés, J.F., (eds.), La teoría del conocimiento de Leonardo Polo, Pamplona: Eunsa, 2018, 233-239.

Murillo, J.I., Intencionalidad, causalidad y finalidad, en Zorroza, I., Sellés, J.F., (eds.), La teoría del conocimiento de Leonardo Polo, Pamplona: Eunsa, 2018, 273- 287.

Polo, L., Antropologia trascendental, I. La persona humana, en Obras Completas, Serie A, vol. XV, Pamplona: Eunsa, 2015.

Polo, L., Conversaciones en Torreblanca (Colombia), pro manuscripto, 1996.

Polo, L., Curso de psicología general, en Obras Completas, Serie A, vol. XXI, Pamplona: Eunsa, 2018.

Polo, L., Curso de teoría del conocimiento, vol. I, en Obras Completas, 
Serie A, vol. IV, Pamplona: Eunsa, 2015.

Polo, L., Curso de teoría del conocimiento, vol. II, en Obras Completas, Serie A, vol. V, Pamplona: Eunsa, 2015.

Polo, L., Curso de teoría del conocimiento, vol. III, en Obras Completas, Serie A, vol. VI, Pamplona: Eunsa, 2015.

Polo, L., El acceso al ser, en Obras Completas, Serie A, vol. II, Pamplona: Eunsa, 2015.

Polo, L., El conocimiento del universo físico, en Obras Completas, Serie A, vol. XX, Pamplona: Eunsa, 2015.

Polo, L., El Ser I, en Obras Completas, Serie A, vol. III, Pamplona: Eunsa, 2015.

Polo, L., Epistemología, creación y divinidad, en Obras Completas, Serie A, vol. XXVII, Pamplona: Eunsa, 2015.

Polo, L., Filosofía y economía, en Obras Completas, Serie A, vol. XXV, Pamplona: Eunsa, 2015.

Polo, L., Introducción a la filosofía, en Obras Completas, Serie A, vol. XII, Pamplona: Eunsa, 2015.

Polo, L., La esencia del hombre, en Obras Completas, Serie A, vol. XXIII, Pamplona: Eunsa, 2015.

Polo, L., La originalidad de la concepción cristiana de la existencia, en Obras Completas, Serie A, vol. XIII, Pamplona: Eunsa, 2015.

Polo, L., Nominalismo, idealismo y realismo, en Obras Completas, Serie A, vol. XIV, Pamplona: Eunsa, 2016.

Polo, L., Persona y libertad, en Obras Completas, Serie A, vol. XIX, Pamplona: Eunsa, 2017.

Polo, L., Presente y futuro del hombre, en Obras Completas, Serie A, vol. X, Pamplona: Eunsa, 2015.

Sanguineti, J.J., Aspectos del conocimiento intencional según Leonardo Polo, en Zorroza, I., Sellés, J.F., (eds.), La teoría del conocimiento de Leonardo Polo, Pamplona: Eunsa, 2018, 15-37.

Sellés, J.F., ¿Es trascendental la antropología de Viktor E. Frankl?, Madrid: Ápeiron, 2016.

Sellés, J.F., El intelecto agente y los filósofos. Venturas y desventuras del supremo hallazgo aristotélico sobre el hombre (I), ss. IV a. C. - XV, Pamplona: Eunsa, 2012; (II), ss. XVI-XVII, Pamplona, Eunsa, 2017; (III), ss. XVIII-XXI, Pamplona: Eunsa, 2017. 
Sellés, J.F., Intuición y perplejidad en la antropología de Scheler. Introducción, textos, glosas. Cuadernos de Anuario Filosófico, Serie Universitaria, $\mathrm{n}^{\circ}$ 216, Pamplona, Servicio de Publicaciones de la Universidad de Navarra, 2009.

Sellés, J.F., La antropología trascendental de Maurice Nédoncelle, Madrid: Ápeiron, 2015.

Sellés, J.F., The Antropological Foundation of Ethics and its Dualities, en Journal of Polian Studies, 1 (2014) 47-77.

Tomás de Aquino, De Potentia, en Corpus Thomisticus, en https://www. corpusthomisticum.org/

Yepes, R., Leonardo Polo y la historia de la filosofía, en Anuario Filosófico, XXV/1 (1992) 101-124.

Juan Fernando Sellés es profesor en la Universidad de Mavarra

Lineas de investigación:

Leonardo Polo; Santo Tomás de Aquino; Antropología; Filosofía medieval

Publicaciones recientes:

- «Francisco Arrentino, o si se puede ser en gnoseología más escotista que Escoto", Liceo franciscano: revista de estudio e investigación, 70/214 (2020), 101-120

- «La personalidad de Leonardo Polo», Studia Poliana, 22 (2020),15-33

Dirección electrónica: jfselles@unav.es 
